\title{
A C.A.D SYSTEM INTEGRATING ARCHITECTURAL KNOWLEDGE
}

\section{S. HANROT, P. QUINTRAND, J. ZOLLER (GAMSAU)}

E. CHOURAQUI,P. DUGERDIL, P. FRANCOIS, M. RICARD (GRTC)

C. GIRAUD (IIRIAM)

\section{Abstract}

The aim of this project is to tackle the problem of architect's knowledge representation. Our approach is based on the belief that part of architect's knowledge can be extracted from graphical documents and classical vocabulary.

We propose an organisation of that knowledge through different points of views: form, function, construction and different levels of description.

An implementation of this approach is developped in an object oriented programming language under Prolog (Objlog) interfaced with a Smalltalk-80 environnement to handle geometry 


\section{1) INTRODUCTION}

L'analyse des systèmes de CAO en architecture(1) montre que leur utilisation ne se fait que dans les phases finales du projet . les systèmes matériels et logiciels apparaissent comme des machines à dessiner ou, pour les plus periormants, comme machine à instrumenter les projets, c'est-à-dire outils de mise au point et d'achèvement.

Un des problèmes majeurs auquel se trouvent confrontés les créateurs de systèmes concerne les conditions d'accès de l'utilisateur à l'espace informatique afin que l'usage de la machine s'inscrive naturellement dans la pratique de conception. C'est-à-dire qu'il permette à l'utilisateur d'élaborer ses propres stratégies de travail et favorise l'interaction homme/machine par un bon fonctionnement du processus de simulation/communication (2) en exploitant dans les meilleures conditions possible l'espace graphique. Pour cela il faut (3):

- Que la communication entre les deux acteur (homme et machine) soit effective, notamment par la compréhension aisée des codes utilisés.

- Que l'effet de la communication ,transfert d'information, soit productif de nouvelles informations chez les deux acteurs. A chaque étape du processus il y a non seulement "héritage" de valeurs de l'étape précédente, mais aussi production d'un nouvel état des problèmes par transformation de l'information.

Pour satisfaire la première condition le système doit offrir à l'utilisateur des moyens de dialogues exploitant le langage de l'architecte. La restitution des messages graphiques ou non graphiques doit être compréhensible et directement opératoire pour une nouvelle action au gré de l'utilisateur.

Pour satisfaire la seconde le système doit disposer de fonctions de manipulation et de restitution de l'information intégrant une part du "savoir du concepteur" pouvant aider et ou orienter le processus de conception.

En d'autres termes le système doit être "intelligent". Ses fonctionalités peuvent être mises en oeuvre aujourd'hui grâce aux techniques de programmation de l'intelligence artificielle (4).

TECTON se donne comme objectif d'apporter une réponse à ces problèmes .

L'approche intelligence artificielle n'y est pas abordée en terme classique de création d'un "système expert" qui a pour objectif essentiel de représenter et de manipuler des modes de raisonnement ; il ne s'agit pas de construire une machine à résoudre les problèmes architecturaux.dont on connait la complexité, mais plutôt de créer un outil intégrant une part du savoir architectural relatif à la description et à la manipulation des objets architecturaux en cours de conception.

Pour rendre capable l'ordinateur de comprendre le langage de l'architecte, l'univers architectural est appréhendé comme un univers régulier qui peut être décrit, modélisé, représenté. Cette discipline implique une recherche de la pertinence des objets manipulés dans le projet d'architecture. La représentation de ce savoir "pertinent" et "opératoire" exploite notamment des représentations dites "orientés-objets" off́rant un environnement de programmation permettant de créer un univers d'objets structurés $(5,6,7,8)$.

On peut à partir d'une information incomplète sur un objet reconstituer l'information manquante ou du moins explorer le champ du possible c'est-à-dire travailler sur des objets incomplètement décrits pour les définir au fur et à mesure de l'avancement du projet et retrouver en situation informatique les conditions de travail de la situation naturelle. La représentation de la connaissance en intelligence artificielle est de pouvoir gérer notamment le "processus d'héritage" évitant de devoir redéfinir à chaque niveau conceptuel l'ensemble des informations décrites dans le niveau supérieur ; chaque niveau de simulation étant informé par le niveau supérieur par un processus d'héritage de mesures.

Trois classes de problèmes sont abordés dans le projet TECTON :

1 - La modélisation du savoir architectural

2 - La représentation symbolique du savoir architectural

3 - La représentation graphique du savoir architeciural.

\section{2) CORPUS DE MODELISATION ARCHITECTURALE}


L'univers de connaissance en architecture

L'univers des connaissances en architecture est décrit à partir d'une observation des savoirs et des savoirs faire, renvoyant à un ensemble d'objets et de méthodes utilisés dans le projet d'architecture. lis nous sont révélés par les théories, les pratiques et la juridiction.

L'objet architectural en cours de conception est vu au travers du projet comme un assemblage d'objets qui le composent. Le contexte problèmatique et l'ergonomie des modes de représentation renvoient à une description non ambigüe des objets à différents niveaux de définition. Ces derniers correspondent aux différents états d'avancement du projet et aux échelles graphiques de représentation.

Enfin, le vocabulaire architectural et les nécessités de la communication sur le chantier, visant notamment à règler les problèmes d'hétérogénéité technologique, nous offrent une description très précise des limites des objets et des règles de mise en relation des objets entre eux (règne, aplomb, alignement...)(8).

Les ensembles du vocabulaire

Intuitivement, on reconnaît dans le vocabulaire architectural trois grands ensembles de notions:

1) Ensemble des objets tangibles comme parties de l'objet architectural:

(bâtiment, édifice, brique, pièce, espace, fondation, mur, percement ...)

2) Ensemble des qualités ou des relations entre les objets ou des entitées abstraites et qui s'accordent à un point de vue particulier :

(axe, tracé, limites, parties, résistance thermique, charges-surcharges, échelle, coût ...)

3) Ensemble des modes de représentation des objets tangibles : graphiques, solides et symboliques :

(plans, géométral, schématique, maquette, descriptifs ...)

Le corpus de modélisation gère les notions contenues dans ces trois ensembles et vise à établir les régularités de la connaissance architecturale héritée de l'histoire d'un savoir faire et de sa production d'objets tangibles.

\section{Les niveaux de définition}

L'objet architectural est composé de deux sous-ensembles caractèristiques d'objets tangibles : les objets matériels, et les objets vides .

Ces objets peuvent être répartis en quatre classes distinctes organisées en quatre niveaux hiérarchiques selon le principe suivant:

Les objets matériels d'un niveau de définition donnés sont définis comme étant une composition d'un ensemble d'objets du niveau inférieur. Les objets vides ne peuvent être définis qu'à un niveau donné et sont dépendants de l'existance d' objets matériels complémentaires.

Ces niveaux sont correlés à des champs de problèmes spécifiques de la conception architecturale.

Ces couches sont identifiées par des notions profondes du vocabulaire. 


\begin{tabular}{l|l|l|l|} 
NIVEAUX & OBJETS & NONONS & EXEMPLS \\
\hline NIVEAU 1 & $\begin{array}{l}\text { materiel } \\
\text { vide }\end{array}$ & $\begin{array}{l}\text { ensemble } \\
\text { entités urb. paysag. }\end{array}$ & $\begin{array}{l}\text { (edifices, batiments, maisons....) } \\
\text { (rues, places, jardins....) }\end{array}$ \\
\hline NIVEAU 2 & $\begin{array}{l}\text { materiel } \\
\text { vide }\end{array}$ & $\begin{array}{l}\text { division } \\
\text { espaces }\end{array}$ & $\begin{array}{l}\text { (enveloppe, distribution, réseaux...) } \\
\text { (pièces, loggia, entrée...) }\end{array}$ \\
\hline NIVEAU 3 & $\begin{array}{l}\text { materiel } \\
\text { vide }\end{array}$ & $\begin{array}{l}\text { element } \\
\text { percement }\end{array}$ & $\begin{array}{l}\text { (mur, cloison, mobilier...) } \\
\text { (baies, trémies, resevations...) }\end{array}$ \\
\hline NIVEAU 4 & $\begin{array}{l}\text { materiel } \\
\text { vide }\end{array}$ & $\begin{array}{l}\text { constituant } \\
\text { creux }\end{array}$ & $\begin{array}{l}\text { (mortier, briques,profilés...) } \\
\text { (rainure, creux de brique....) }\end{array}$ \\
\hline
\end{tabular}

Le dessin géométral fait appel aux opérateurs de la géométrie et vise par la figure et la cote à produire des représentations de l'objet architectural selon des échelles géometriques. Celles ci permettent d'établir un rapport homomorphique entre l'objet réel et l'objet figuré. Pratiquement, cinq échelles ont été sélectionnées par les concepteurs pour représenter un objet architectural:

$-1 / 500: 2 \mathrm{~mm}$ par-metre ; $1 / 200: 5 \mathrm{~mm} \mathrm{pm} ; 1 / 100: 1 \mathrm{~cm} \mathrm{pm} ; 1 / 50: 2 \mathrm{~cm} \mathrm{pm} ; 1 / 20: 5 \mathrm{~cm} \mathrm{pm}$

II existe une correspondance entre les échelles de représentation géométrales et les niveaux hiérarchiques d'objets tangiblesqui conduisent à établir quatre niveaux de définition de l'objet architectural.

\begin{tabular}{l|l|c|} 
NIVEAUX & NOnONS & ECHELE GEOMETRALE \\
\hline NIVEAU 1 & $\begin{array}{l}\text { ensemble } \\
\text { entités urb. paysag. }\end{array}$ & $1 / 500---1 / 200$ \\
\hline NIVEAU 2 & $\begin{array}{l}\text { division } \\
\text { espaces }\end{array}$ & $1 / 200---1 / 100$ \\
\hline NIVEAU 3 & $\begin{array}{l}\text { element } \\
\text { percement }\end{array}$ & $1 / 100---1 / 50$ \\
\hline NIVEAU 4 & $\begin{array}{l}\text { constituant } \\
\text { creux }\end{array}$ & $1 / 50---1 / 20$ \\
\hline
\end{tabular}

Les champs de définition

A chaque niveau de définition, les objets peuvent être décrits selon différents points de vue. Ainsi, un mur est-il défini comme un organe support (point de vue fonctionnel), mais aussi comme un ouvrage (point de vue constructif), ou comme une forme (point de vue morphologique).

Chacun de ces points de vue confère aux objets un ensemble de propriétés spécifiques. Nous les appelons "champs de définition".

Exemple de représentation du mur en tant qu'élément de niveau de défínition 3 


\begin{tabular}{|c|c|c|c|c|c|}
\hline OBJET & & statique & thermique & ructi & (5) \\
\hline mur & $\begin{array}{l}\text { attributs- } \\
\text { architectoniques : } \\
\text { nu } \\
\text { alignement } \\
\text { aplomb }\end{array}$ & resistance & coef $k$ & $\begin{array}{l}\text { coulé } \\
\text { banché } \\
\text { maçonné }\end{array}$ & $\begin{array}{l}\text { classique } \\
\text { high-tech } \\
\text { rustique }\end{array}$ \\
\hline
\end{tabular}

Les relations entre objets

Parmi le grand nombre des relations concevables entre les objets, nous retenons principalement les relations de calage et de composition, mal gérées par les systèmes de CAO existants et pourtant très opératoires et fondamentales dans la pratique courante.

Ces relations mettent en oeuvre différents points de vues. De la sorte, des contraintes et des controles peuvent être gérés par des règles particulières.

\section{LE CALAGE}

Cette relation est mise en oeuvre dans le projet pour positionner les objets tangibles entre eux et avec des êtres architectoniques (axes , trames,...).

Pour représenter cette relation, nous distinguerons trois sous types de relations de calage:

- Les primitives de calage (mise au nu, mise à l'axe...)

- Les configurations de calage (chainage d'angle droit...)

- Les chaines de calage. (ensemble d'objets dépendants)

\section{LACOMPOSITION}

La composition consiste à ordonnancer des objets selon un ensemble de règles, et à décréter que cet ensemble d'objets constitue un objet plus complexe.

Inversement, un objet composé est suceptible d'être decomposé en un ensemble ordonancés d'objets moins complexes, parties de la composition .

Ainsi une colonnade est un objet composé de colonnes et d'un entablement selon des règles d'espacements des entraxes, relatives à la portée des ouvrages, à la stylistique... .

\section{HIERARCHISATION DES RELATIONS}

Une relation de composition peut être ramenée à un ensemble de relations de calage additionnée d'un ensemble de règles. Les relations de calage peuvent elles mêmes être ramenées à un ensemble de primitives géométriques et à un ensemble de règles .

II existe donc une hiérachie entre ces relations et une inclusion des unes dans les autres: 


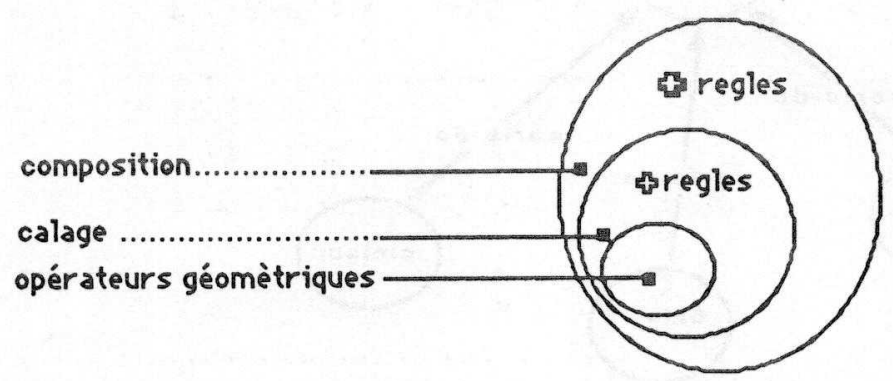

\section{3) LA REPRESENTATION SYMBOLIQUE DU SAVOIR ARCHITECTURAL}

Le modèle orienté objet fournit aux architectes un cadre conceptuel de réflexion et induit une méthodologie de catégorisation de leur savoir. Ainsi un outil de représentation informatique peut-il être défini en essayant de s'adapter au mieux à ce découpage cognitif

L'élément fondamental est l'objet, qui se répartit en deux types : les classes et les instances de classes. Les classes représentent les connaissances architecturales. Elles contiennent en intention ou en extension toutes les informations requises pour générer un objet élémentaire d'un projet architectural représenté par une instance de classe.

Les classes et les instances de classes sont formées de champs ('slots') eux-même constitués d'aspects ('facets'). Les champs sont les éléments de description des objets. Les aspects sont les traits sémantiques des champs. On distingue deux types d'aspects : les aspects statiques et dynamiques. Les aspects statiques décrivent en intention ou en extension l'ensemble des valeurs possibles pour le champ. Les aspects dynamiques contiennent des procédures (démons) qui permettent de propager les contraintes dans la base de connaissance à la suite d'une manipulation de champ. Deux sortes de champs sont distingués dans notre modèle : les champs à valeur terminale ou atomique (de type prédéfini : entier, réel, identificateur ou chaine de caractères) et les champs à valeur référentielle (pointeur sur des objets). Ces derniers représentent des relations entre les objets. Les champs des instances de classes contiennent des valeurs qui doivent être compatibles avec les déclarations des classes.

Le mécanisme fondamental d'exploitation de ce type de représentation est l'héritage. II permet de décrire un objet architectural donné en plusieurs niveaux conceptuels. Ces niveaux sont matérialisés par un graphe dont les noeuds sont des classes et les arcs une relation d'ordre ("sorte-de"). L'héritage est un mécanisme de recherche d'information qui consiste à accéder, à partir d'une classe du graphe, à toutes les classes suivantes. Les informations contenues par ces dernières sont considérées comme faisant également partie de la classe de départ. Dans notre modèle, nous avons étendu ce principe à des relations $n$-aires. C'est-à-dire qu'un objet peut accéder simultanément aux informations de plusieurs objets de niveau supérieur (héritage multiple). De plus, nous avons défini un nouveau type d"héritage entre objets, baptisé "héritage sélectif",qui permet à un objet représentant une partie d'un objet composite, d'accéder sélectivement à un sous-ensemble des déclarations de l'objet composite associé. Cet héritage et la relation associée ("partie-de") se sont avérés très intéressants pour modéliser la décomposition d'objets architecłuraux complexes en objets plus simples. La cohérence des déclarations est alors automatiquement assurée au moyen de l'héritage .

Illustration de l'économie des déclarations de propriétés introduite par les mécanismes d'héritage vertical (relation "sorte-de") et sélectif (relation "partie-de"): 


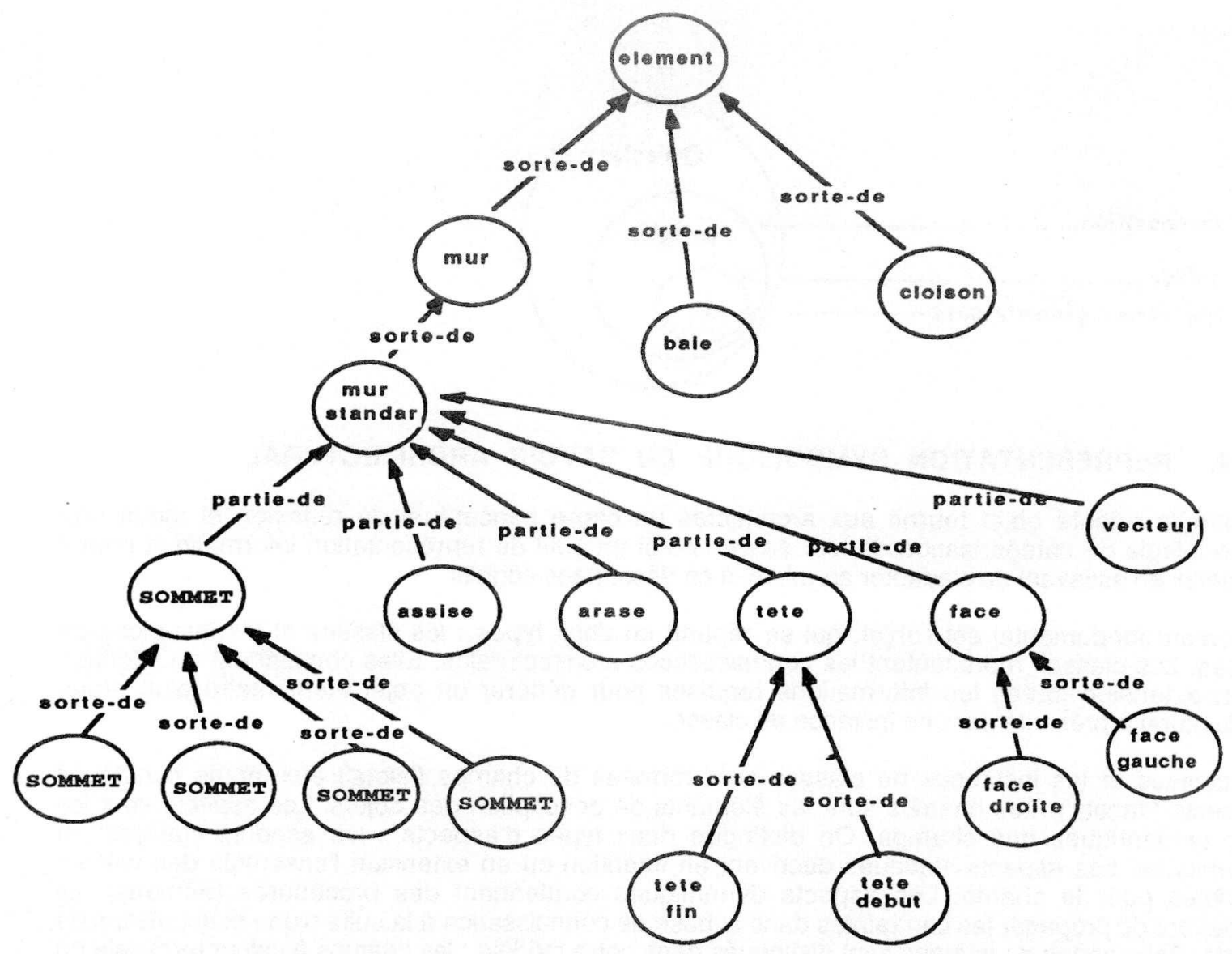

graphe d'une base de connaissances avec héritages

Afin de compléter notre modèle, nous avons introduit un mécanisme de communication entre objets sous forme d'envoi de messages. Chaque objet est alors considéré comme une entité indépendante capable d'effectuer de manière autonome un certain nombre d'action suite à une demande formulée par un message. Ce système, assez répandu dans ce type de représentation, permet d'élever le niveau d'abstraction auquel le concepteur d'une base de connaissance travaille. En effet, toutes les manipulations sur les objets de l'univers décrit peut se traduire sous forme d'envoi de messages, sans se préocuper de la façon dont l'objet récepteur traitera la requête. Seul importe la réaction attendue de l'objet.

Ce modèle a été implémenté sous forme d'un langage orienté objet au dessus de Prolog. II permet aux architectes de tester la pertinence du modèle proposeé vis à vis des problèmes posés par la formalisation de leur savoir. 


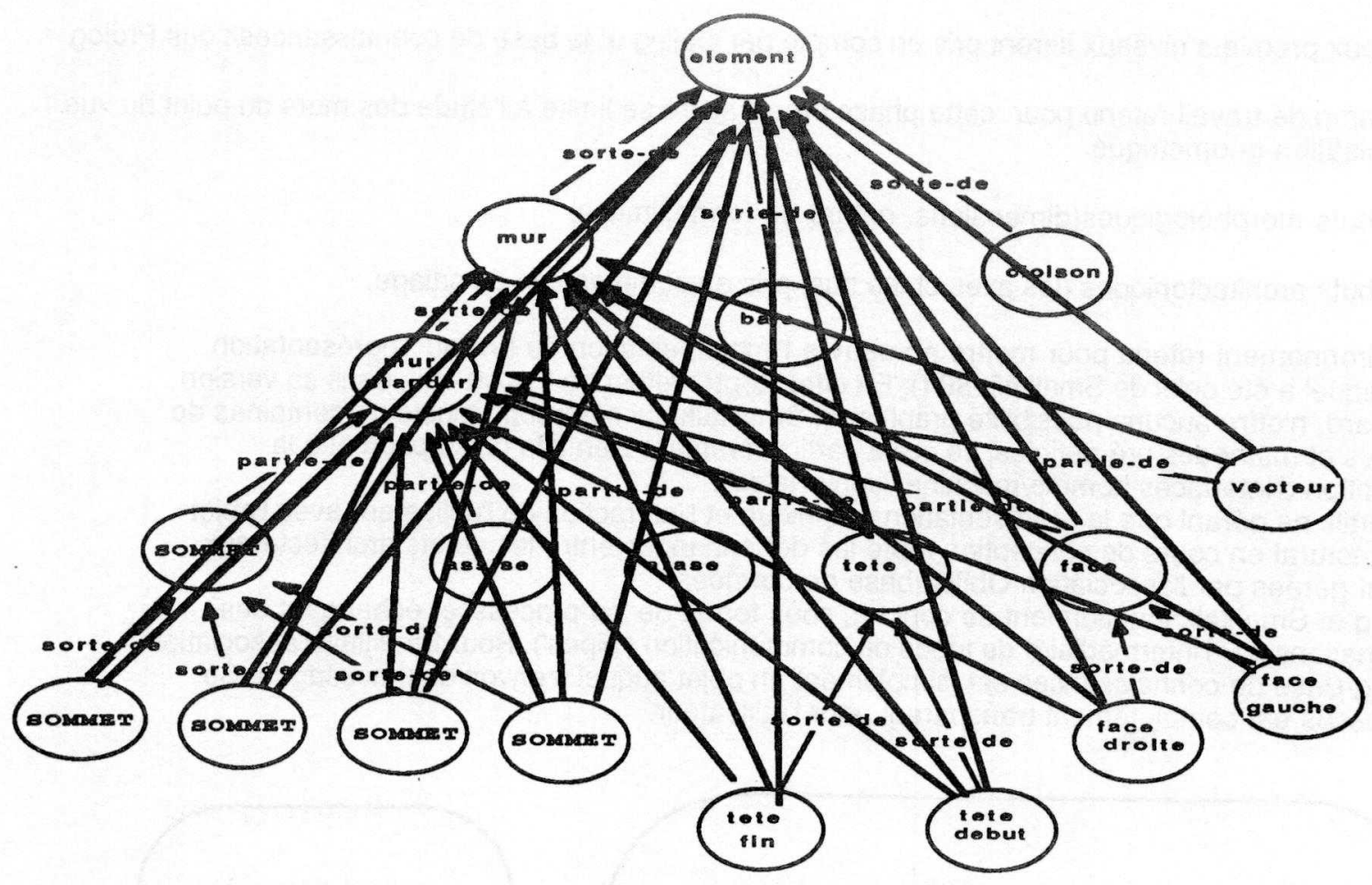

graphe de la même base de connaissances sans les héritages.

\section{4) LA MODELISATION GEOMETRIQUE}

Les objectifs du projet TECTON en matière graphique sont de denner à l'architecte un outil de conception qui intégre les techniques d'intelligence artificielles et permette :

- La manipulation des formes géométriques, la définition et la modification de leurs relations

- La représentation graphique des objets sur les périphériques de visualisation

- L'interaction avec le modèle conceptuel de l'objet, aussi bien pour décrire que modifier, à partir du niveau graphique, tous les types de connaissances qui le concerne.

Pour atteindre cel objectif, nous distinguons les niveaux de modélisation géométrique suivants :

- Un niveau purement conceptuel dans lequel la composante géométrique des objets est décrite par ses propriétés et par ses relations

- Un niveau formel d'expression de contraintes, spécialisation du niveau précédant. Ces contraintes résultent de la nature géométrique des objets ainsi que de leurs assemblages.

- Un niveau de représentation graphique, qui est une solution à chaque instant du modèle précédant en terme de modèle tridimensionnel classique $\mathrm{De}$ ce modèle on peut extraire des vues bidimensionnelles visualisables sur un périphérique graphique et susceptible de servir de support à la manipulation interactive de l'objet. 
Les deux premiers niveaux seront pris en compte par Objlog et la base de connaissances sous Prolog.

Le champ de travail retenu pour cette phase préparatoire se limite á l'étude des murs du point du vue modélisation géométrique

- Attributs morphologiques(dimensions, nature de forme,limites)

- Attributs architectoniques (les axes et les nus) pris en comptes lors du calage.

L'environnement retenu pour mettre en oeuvre l'implémentation du niveau 'représentation graphique' a été celui de Smalltalk-80 (). En effet, contrairement à Prolog qui , dans sa version standard, n'offre aucune possibilité graphique, Smalltalk qui comprend plusieurs centaines de classes et méthodes pré-définies, se prête particulièrement bien à la conception et à la réalisation d'interfaces homme/machine 'conviviales' .

Smalltalk ne gérant que la représentation graphique et l'interaction de l'utilisateur avec l'objet architectural en cours de conception toute les dépendances entre les objets architecturaux seront gérées par l'association Objlog/base de données.

Prolog et Smalltalk fonctionnent de concert, sous forme de 'co-process' et échangent des informations par l'intermédiaire de tubes de communication ('pipes'). Pour Smalltalk l'association Objlog/Base de connaissances est simplement un objet auquel il envoit des messages. Ce processus est complétement transparent pour l'utilisateur.

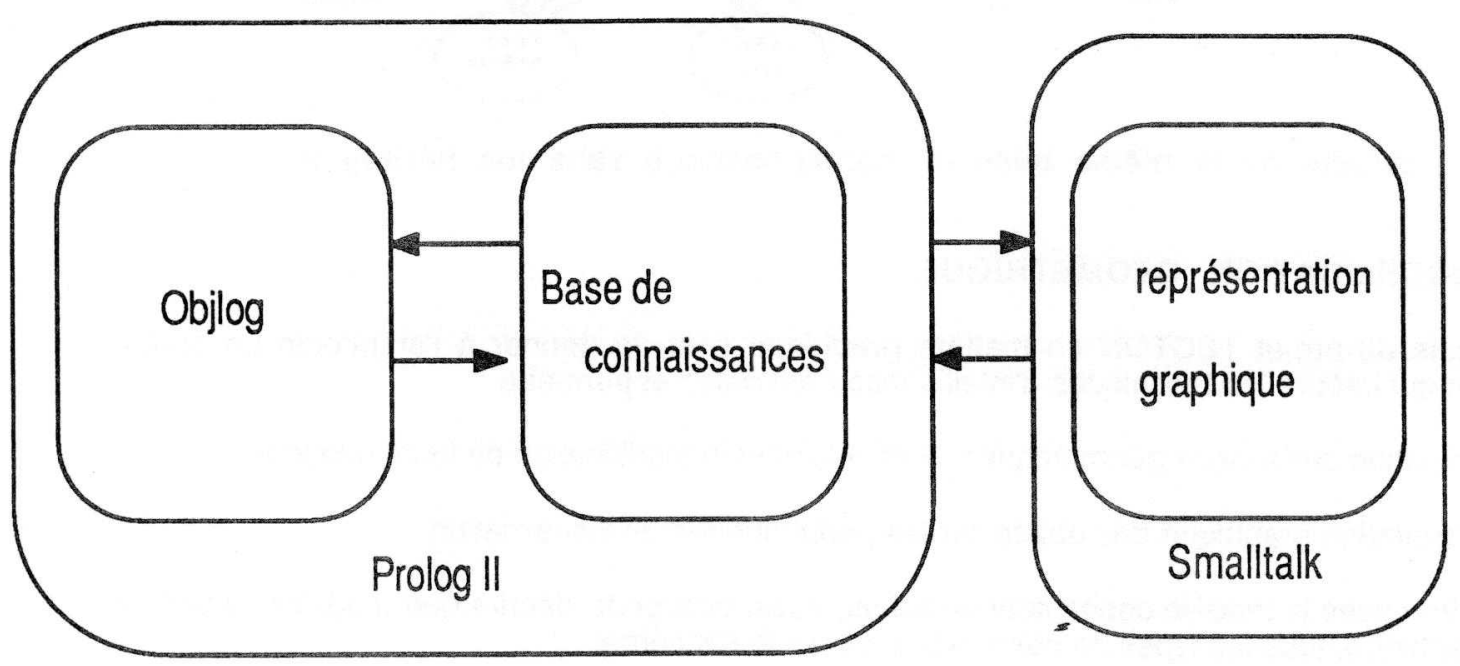

Notre attention s'est pontée essentiellement sur l'interaction de l'uitilisateur avec l'objet architectural en cours de conception par lintermédiaire de ses representations graphiques

Pour cela nous avons utilisé la triade modèle - vue - contróleur, un concept mis en oeuvre en Smalltalk:

Triade modèle - vue - contrôleur

Un des problèmes des logiciels de CAO classique réside dans l'etroite imbrication entre le modèle, la visualisation et l'interaction. Smalltalk distingue ces trois éléments et permet de les lier de telle façon qu'une action sur la vue associée au modèle, est prise en compte par le contrôleur qui envoie au modèle les messages nécessaires à sa mise à jour .

- Le "Modèle" représente les données du problème ainsi que les opérations qui y sont attachées. 
- La "Vue" présente à l'utilisateur les informations provenant du modèle (en général par l'intermédiaire de l'écran)

- Le "Contróleur" interpréte les "entrées" provenant de l'utilisateur (par l'intermédiaire du clavier ou de la "souris") et envoie au modèle les messages de mise à jour correspondants.

Cette modélisation se prête particuliérement bien à notre problème :

- Le 'modèle' est un objet représentant l'ensemble du projet architectural.

- La 'vue' la représentation sur un terminal graphique (écran ou traceur) de l'ensemble ou d'une partie du projet

- Le 'contrôleur' autorise l'interaction de l'utilisateur avec le projet par l'intermédiaire de périphériques de saisie permettant de pointer un objet architectural particulier sur la vue qui lui est associé, ou de choisir une commande dans un menu déroulant ( mode d'action particulier : saisie d'axes, repérage d'un mur ...).

Pour déclencher la mise a jour des vues qui lui ont été associées, le modèle utilise la dépendance ( changed, update ). Pour un même modèle on peut avoir autant de doublet vue - contrôleur que nécessaire ( ex: plan, vue de face , vue de profil, vue tridimensionnelle .......)

\section{CONCLUSION}

- Ce projet en fin de première phase apporte les éléments de validation suivants aux hypothèses de départ

a) la pertinence de l'identification et du découpage de l'univers architectural (vocabulaire architectural et objets de représentation)

b) le modèle choisi : découpage par points de vue et par niveaux

c) la possibilité de représenter le savoir architectural dans l'environnement orienté objet l'héritage sélectif.

d) la pertinence de l'outil informatiqued développé pour gérer les héritages notamment

e) La pertinence d'un modèle géométrique orienté objet pour la représentation de l'objet architectural et la gestion de l'interaction de l'utilisateur/modèle en phase de conception.

II propose une implémentation d'un sous ensemble restreint qui sera soumise à une expérimentation sous un cadre opérationnel incluant notamment : une modélisation des principaux concepts du savoir architectural dans une première version de l'environnement informatique constitué dans un langage orienté objet et des outils graphiques de représentation et de communication (H/M).

- Une deuxième phase permettra à terme (fin 88 ) de propose une maquette démonstrative du système portant sur un sous ensemble de l'univers de connaissance architecturale (univers des Murs, Baies et Couvertures). 


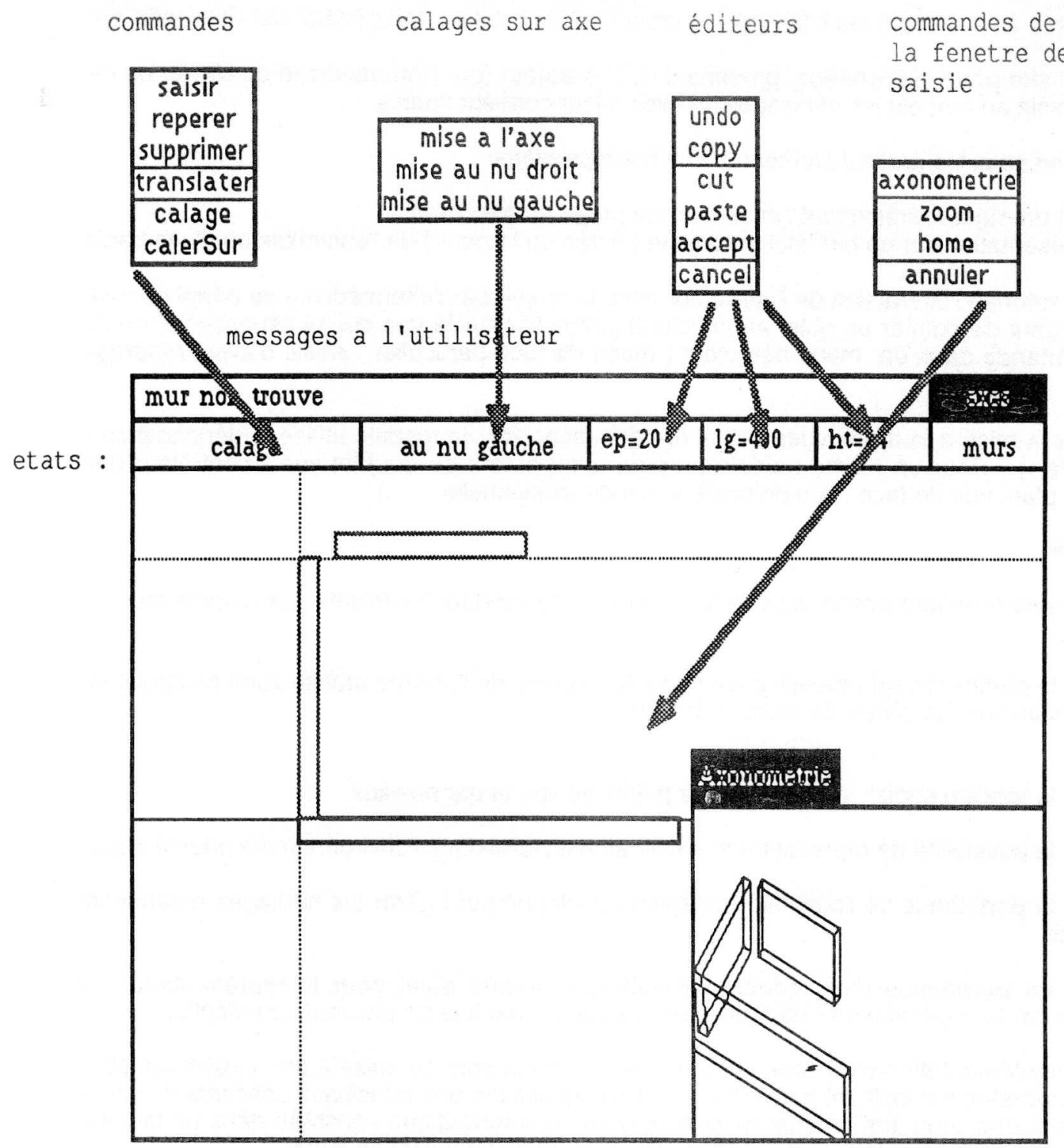

exemple de vue associée à un modèle

\section{BIBLIOGRAPHIE}

(1) LEBAHAR J.C.1985 Etudes en CFAO, Hermes.

(2) LEBAHAR J.C.1983. Le dessin d'architecte. Parenthèse, Marseille.

(3) FREGIER M. 1984. Etude de la représentation des composants morphologiques des données du projet architectural dans un contexte de CAO. GAMSAU/Plan construction. 1984. 
(4) GIRAUD C. janv.984. La CAO. Interface-Afcet.

(5) QUINTRAND P. Travaux GAMSAU sur la régularité en architecture. Identification des composants architecturaux et constructif manipulés dans le projet d'architecture. GAMSAU/BETEREM/Plan construction. 1985.

(6) CHOURAQUI E., DUGERDIL PH. - Application des langages orientés à la CAO de l'architecture.Actes des 3èmes Journées d'Etude sur les langages orientés objet de L'AFCET, Paris, 8-10 janvier 1986

(7) DUGERDIL PH. - Une méthodologie orientée objet pour la représentation des connaissances en CAO architecture. Mémoire de DEA en Informatique et Mathématiques, Faculté de Luminy, univ. d'AixMarseille II, 1985.

(8) HANROT S. -Elements de modèlisation des connaissances architecturales. Memoire de DEA en informatique mention XIAO, Faculté de ST-Jerome, Univ. AIX-Marseille III 1986.

(10) BOBROW D.G., WINOGRAD T. - An overview of KRL, a knowledge representation language. Cognitive Science, Vol $1 \mathrm{~N}^{\circ} 1,1977$.

(11) GOLDBERG A. , ROBSON D., Smalltalk-80, the language and it implementation, Addison Wesley, 1983 